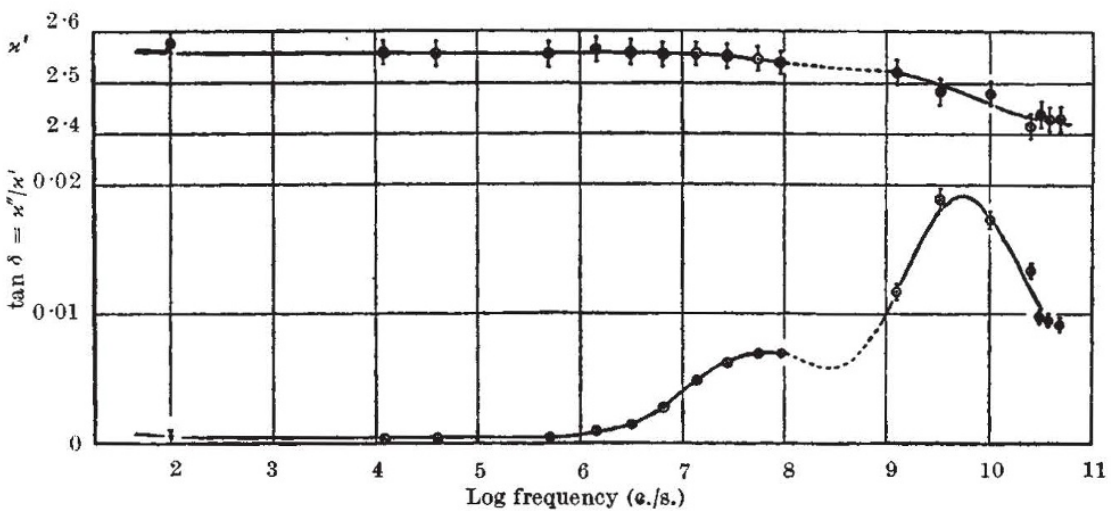

the frequency of each violin varies in random fashion under various influences, especially the pressure of the bow.

(2) The customary vibrato playing itself modifies continuously both the frequency and amplitude of the note, thus preventing the ear from perceiving regular beats between the two violins.

Experiment shows that the complex note produced by each instrument gives rise in an extended medium

I wish to thank the staff of the Electrical Research Association for their assistance in preparing the purified material to which the present measurements relate, and Dr. J. A. Saxton, of the Department of Scientific and Industrial Research, for the measurement at $5 \times 10^{10} \mathrm{c} . / \mathrm{s}$.

Electrical Engineering Department,

\section{WILLIS JACKSON}

Imperial College of Science and Technology, London, S.W.7.

Aug. 1.

${ }^{1}$ Dryden, J. S., and Jackson, W., Nature, 162, 656 (1948).

\section{An Interference Paradox in Sound}

ONE of the commonest demonstrations of interference is that between two loud-speakers actuated by separate audio-frequency oscillators. When the two oscillators differ slightly in pitch, the audience perceives beats which form a complicated pattern of interferences in the room. At any point in the room there is a probability of $\frac{1}{2}$ that the phase difference of waves coming from the two sources lies between $\pi$ and $2 \pi$, consequently that the sound-level may be less than that due to one source alone, a fact that is readily demonstrated with a microphone.

This conclusion could be extended to cover any case of two sources in consonance, for example, to two violins playing in unison. Put in the form of this paradox: "Do two violins make more sound than one alone ?", the question may give rise to confusion in the minds of those who favour a negative answer on the basis of the theory of interference, as it is usually taught, yet whose experience is at variance with such a conclusion. Anyway, if the two violins are not exactly in unison, beats should be heard which should be perceived by the ear, even if the ear is not sensitive to local variations in sound-level.

I suggest that in addition to obvious causes such as the sources not being points or remaining stationary, other causes operate to make two violins always sound louder than one :

(1) Slight variations of the pitch of each violin round about their nominal value occur. Besides the inability of a player to hold an instrument in precise intonation due to human failing, oscillograph records of the sounds of a violin show objective changes of pitch with the tension, which tends to decrease, especially after pizzicati, as the strings relax. This is most marked with catgut but still noticeable with metal strings, the repeated stresses leading to relaxation of the pegs in their holes and a drop in frequency which may amount to 2 per cent. Besides this shift, to a resultant note still more complex, the amplitude of which varies in a scarcely perceptible fashion by not more than a few decibels. Any notion of rhythm in these variations, so important for the clear perception of beats, is completely wanting. Sometimes at low frequencies, however, a series of five or six successive beats may be heard.

(3) In a room another phenomenon is superposed. According to a theorem of Rayleigh, when an increasing number of waves from sources of random phase distribution are superposed, the pressure amplitude increases as the square root of their number, approximately, a case which should apply to an orchestra. When there are two sources only, one must still consider their acoustic 'images' formed in the walls of the room, so that the effect at any point in the room may still be regarded as that of a number of sources of random phase distribution. In practice, due to absorption, only the reflexions in the walls of first orders need be considered.
Laboratoire d'Acoustique,
66 rue Saint Sebastien,

\section{A. Moles}

$$
\text { Marseilles. }
$$

\section{Disintegration of Helium by $\mathrm{\Pi l}^{-}$Mesons}

MANY estimates have been made of cross-sections for meson capture; but apparently the resulting nuclear disintegrations have seldom been treated. Observations on the energies of the emitted particles might give some information on the interaction between mesons and nucleons and would show whether a neutral meson is produced or the whole meson mass is absorbed. Experimental results could be interpreted without ambiguity only in the case of very light elements. Helium is the lightest element to emit a charged particle and might be used in experiments with artificially produced mesons.

We have calculated the energy spectra and relative probabilities of the three ways of disintegration of helium-4 on the following assumptions: the meson is captured from the lowest Bohr orbit; the whole mass (290 electron masses) is converted into energy ; the interaction energies are $(a) \psi(r)$ or $(b) \sigma \cdot \nabla \psi(r)$, where $\psi(r)$ is the scalar meson wave function at the position of a proton and $\sigma$ is the spin operator of the proton. Gaussian wave functions $\left(\exp \left(-\alpha \sum r_{i j}^{2}\right)\right.$, $1 / \sqrt{ } \alpha=4.0 \times 10^{-13} \mathrm{~cm}$.) are used for the nuclei and plane waves for the emitted particles. The transition probabilities are determined by the usual perturbation method. 